\title{
Model photo-responsive elastomers based on the self-assembly of side group liquid crystal triblock copolymers (Presentation Recording)
}

\author{
Zuleikha Kurji, Washington State Univ. (United States); Julia A. Kornfield, California Institute \\ of Technology (United States); Mark G. Kuzyk, Washington State Univ. (United States)
}

\begin{abstract}
We report the synthesis of azobenzene-containing coil-liquid crystal-coil triblock copolymers that form uniform and highly reproducible elastomers by self-assembly. To serve as actuators to (non-invasively) steer a fiber optic, for example in deep brain stimulation, the polymers are designed to become monodomain "single liquid crystal" elastomers during the fiber-draw process and to have a large stress/strain response to stimulation with either light or heat. A fundamental scientific question that we seek to answer is how the interplay between the concentration of photoresponsive mesogens and the proximity to the nematic-isotropic transition governs the sensitivity of the material to stimuli. Specifically, a matched pair of polymers, one with $\sim 5 \%$ azobenzene-containing side groups ( $\sim 95 \%$ cyanobiphenyl side groups) and the other with $100 \%$ cyanobiphenyl side groups were synthesized from identical triblock pre-polymers (with polystyerene end blocks and 1,2-polybutadiene midblocks). These can be blended in various ratios to prepare a series of elastomers that are precisely matched in terms of the backbone length between physical crosslinks (because each polymer is derived from the same pre-polymer), while differing in \% azobenzene side groups, allowing the effect of concentration of photoresponsive groups to be unambiguously determined.
\end{abstract}

View presentation video on SPIE's Digital Library: http://dx.doi.org/10.1117/12.2186347.4519371759001 